
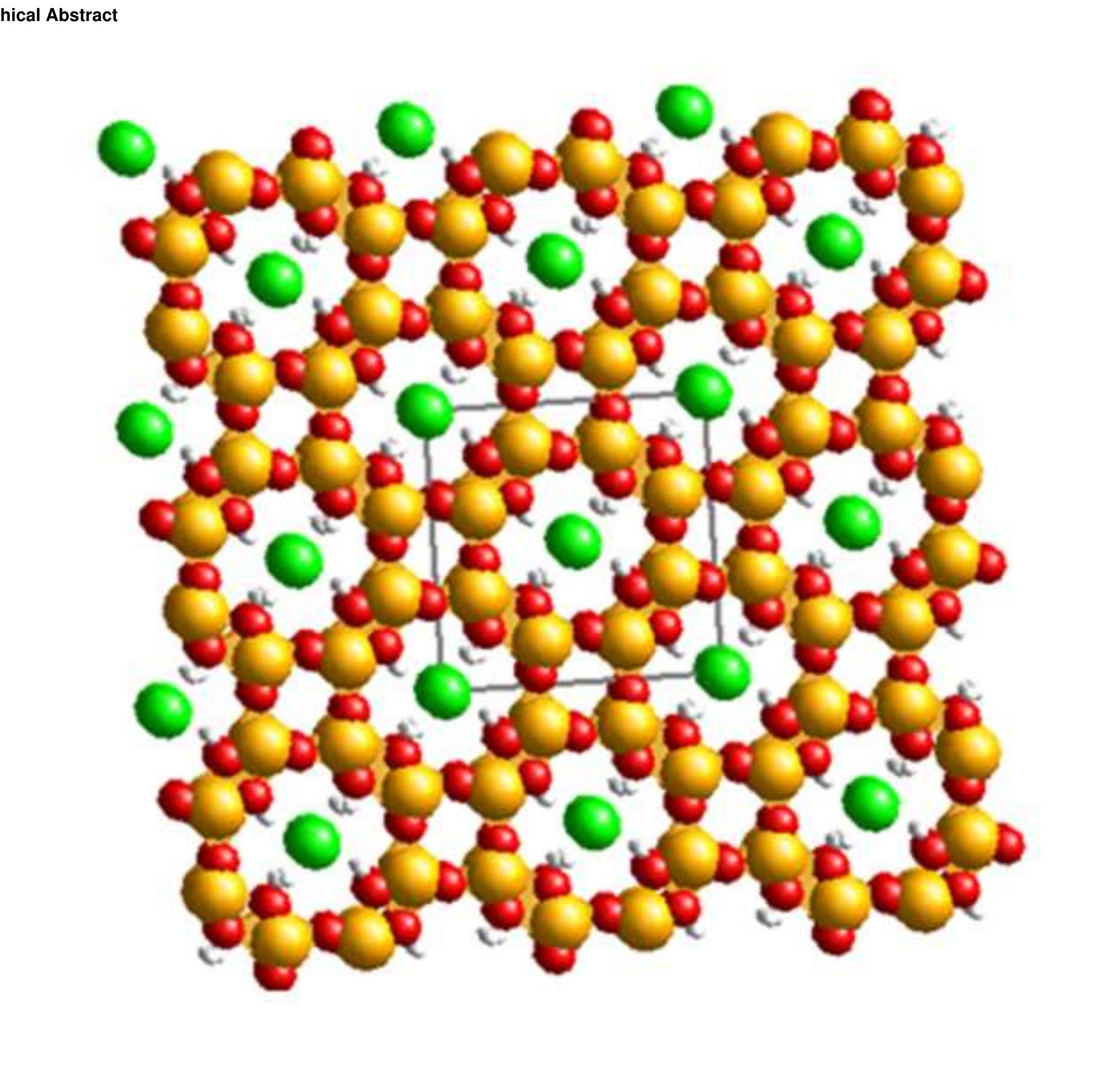


\title{
Synthesis, characterization and thermodynamic study of carbon dioxide adsorption on akaganéite
}

\author{
R. Roque-Malherbe ${ }^{*}, 1$ F. Lugo ${ }^{1}$, C. Rivera-Maldonado ${ }^{1}$, R. Polanco-Estrella ${ }^{1}$ and \\ O. Uwakweh ${ }^{2}$ \\ ${ }^{1}$ School of Science, University of Turabo, PO Box 3030, Gurabo, PR, 00778-3030, USA. \\ ${ }^{2}$ Materials Science Department, University of Puerto Rico-Mayagüez Campus, Mayaguez, PR \\ 00681-9044, USA. 787-832-4040
}




\begin{abstract}
A mixture of akaganéite nanoparticles and sodium salts was synthesized and modified, first by washing, and then by Li exchange. The structural characterization of the produced materials was performed with: powder X-ray diffraction, scanning electron microscopy, energy dispersive Xray analysis, thermo-gravimetric analysis, diffuse reflectance infrared Fourier transform spectrometry, Mössbauer spectroscopy and magnetization measurements. Additionally low pressure nitrogen and high pressure carbon dioxide adsorption experiments were performed. The sum of the characterization information made possible to conclude that the produced akaganéite phases crystallized in a structure exhibiting the symmetry of the $I 2 / \mathrm{m}$ space group, where the measured equivalent spherical diameter of the akaganéite crystallites yielded $9 \mathrm{~nm}$, as well, the tested phases exhibited a standard behaviour under heating and displayed a superparamagnetic behaviour. Finally the high pressure carbon dioxide adsorption experiments demonstrated a pressure-responsive framework opening event due to a structural transformation of the adsorbent framework induced by the guest molecules. This fact opens new applications for akaganéite as a high pressure adsorbent.
\end{abstract}

Keywords: carbon dioxide, adsorption, akaganeite, superparamagnetism, gas storage. 


\section{Introduction.}

Iron oxides, oxide hydroxides and hydroxides are common compounds; that, as a consequence of their magnetic [1-4], catalytic [5,6] and adsorption [7,8] properties are some of the most important materials on earth. In particular, the oxide hydroxide akaganéite is an important constituent of soils, geothermal brine deposits and corrosion products [9-21].

The common structural building block for all the known oxide hydroxides is the $\mathrm{Fe}(\mathrm{O}, \mathrm{OH})_{6}$ octahedron organized in different forms [1]. In particular, the octahedrons generating the framework produce a hollandite-type structure displaying the symmetry of the $I 2 / m$ (No. 12) space group; where, four double chains of octahedrons create tunnels with square cross sections [9]. Further, these tunnels are in part occupied by chloride anions whose charges are compensated by protons or positive ions [11,21]. Moreover, the octahedrons are spatially organized to generate two different octahedral sites for the $\mathrm{Fe}^{3+}$ ions to produce antiferromagnetic behaviour, owing to the alignment of their magnetic moments [13]. However, the small size of the crystallites creates a situation where the direction of the magnetic moment of the crystallites turns out to be unsteady and fluctuates, producing superparamagnetism [14]. In addition. akaganéite, on heating, release adsorbed water (ca. $350 \mathrm{~K}$ ); next, a dehydroxilation process takes place (at ca. $480 \mathrm{~K}$ ); finally, an exothermic peak found at ca. $633 \mathrm{~K}$ has been associated to both the loss of $\mathrm{HCl}$ and the beginning of the formation of hematite and in some cases magnetite [12]. One more important feature of akaganéite is the morphology exhibited by their crystals. In this regard, the recognized crystallite forms for this material are somatoids or spindle-shaped and rods or needle-like [15]; even though, additional one-dimensional morphologies, like tubes, and fibres had been as well obtained [16]. Hence, the essential 
morphological characteristic of akaganéite is one-dimensionality. This feature is due to the hollandite type framework, since, the channels run through the [010] direction, accordingly, in this direction the crystal is elongated, meanwhile along the [100] and [001] crystallographic directions the crystal is narrow [10]. As a result, the crystal sizes are normally between 100-1000 $\mathrm{nm}$ along the [010] crystalline direction and 10-100 $\mathrm{nm}$ along the [100] and [001] crystalline directions [1]. Furthermore, as a consequence of their small crystallite sizes akaganéites show a high specific surface area (S), i.e., together with the micropores this material show a developed external surface [5].

After thermal dehydration of akaganéite water molecules are detached; thereafter, a structure showing an open channel system, suitable for the adsorption of small molecules and a relatively high external surface area is produced $[7,8]$. Moreover, owing to the existence of a developed external surface akaganéite can as well adsorb ions on this surface [17-20]. In this regard, adsorption of arsenite $[17,18]$ and phosphates $[19,20]$ on the akaganéite outer surface have been investigated, yielding experimental adsorption isotherms which are, in general, fitted with the Freundlich equation [19]. Hence, both gas adsorption in the micropores and ion adsorption on the external surface are important properties of akaganéite.

In the present research we studied carbon dioxide adsorption on a thoroughly characterized akaganeite; since, this molecule is a good probe to study adsorption on microporous materials [22]; given that, it is an excellent tool for the measurement of the micropore volume [23] and adsorption interactions [24]. In particular, in the present case owing to the magnetic properties of akaganeite; if these magnetic properties could be modified by controlling the electronic structure of the akaganéite through adsorbed molecules, this process could produce effects that might be used in practice. Further, adsorption is a useful process for the separation, storage and recovery 
of gases [25]. In particular, the separation, storage and recovery of carbon dioxide are very important research topics; since, it is a reactant in significant industrial processes and a greenhouse gas which contributes to global warming [26].

Consequently, in the research reported here we describe the synthesis, and structural characterization of an akaganéite; together with the investigation of their magnetic and adsorption properties. The research was carried out by measurements and characterizations consisting of powder X-ray diffraction (XRD), scanning electron microscopy (SEM), energy dispersive X-ray analysis (EDX), thermogravimetric analysis (TGA), diffuse reflectance Fourier transform infrared spectroscopy (DRIFTS), Mössbauer spectroscopy (MS) and magnetization experiments; while the adsorption investigation was performed with volumetric adsorption analysers.

\section{Experimental procedures}

\subsection{Synthesis and modification}

All the consumables chemicals were analytical grade without additional purification. The water used in the synthesis process was bi-distilled.

The synthesis was performed by the acid hydrolysis of iron chlorides [16, 27-30], in this manner: one solution of ferrous chloride and ferric chloride 1:2 was prepared, hereby identified as solution A; a second solution of sodium hydroxide 6M was also produced, identified as solution $\mathrm{B}$; solution $\mathrm{B}$ was mixed under agitation with solution $\mathrm{A}$; and stirred for 60 minutes at $20{ }^{0} \mathrm{C}$; then, this mixture was heated at $90{ }^{\circ} \mathrm{C}$ for 30 minutes under stirring conditions; after that, a 0.3 M sodium citrate solution was added; afterwards, the entire resulting mixture was heated at $90{ }^{0} \mathrm{C}$ 
for 30 minutes and the precipitated reaction product washed with acetone and de-ionized water; later, the washed with de-ionized water material was centrifuged three times for 15 minutes at 4000 RPM; to conclude the process, the sample was dried in vacuum without heating.

The complete synthesis procedure was carried out in nitrogen atmosphere at ambient pressure. Additionally, the process was repeated obtaining in both cases the same product, namely the assynthesized sample, labeled AK-AS.

The first modification of the as-synthesized akaganéite was a thorough washing process with bidistilled water to get the washed sample identified as AK-W. Thereafter, this material was exchanged at temperature during $72 \mathrm{~h}$. with a $2 \mathrm{M} \mathrm{LiCl}$ aqueous solution at ratio of $1 \mathrm{~g}$ of sample per one liter of solution, obtaining the Li exchanged sample, identified as AK-Li.

\subsection{Methods}

Firstly, the XRD profiles were gathered with a Bruker D8 Advance system in Bragg-Brentano vertical goniometer configuration. The $\mathrm{X}$-ray radiation source was a ceramic $\mathrm{Cu}$ anode tube. To confirm the assigned structure of the synthesized materials, the gathered XRD patterns were refined with the Pawley method. The computer program used to carry out the Pawley refining processes was the Bruker DIFFRACplus TOPAS ${ }^{\mathrm{TM}}$ software package [31].

Secondly, the SEM study was performed with a JEOL JSM-6360, whose electron beam acceleration voltage was $20 \mathrm{kV}$. As well, the elemental chemical analysis of the as-synthesized and washed samples, were performed using an EDX spectrometer coupled into the microscope. To improve the accuracy five the elemental composition of five spots was measured an calculated the average composition. 
Next, the TGA testing process was carried out with a TA, Q-500 equipment; where the temperature was linearly scanned, from 25 to $600{ }^{0} \mathrm{C}$, at a rate of $5{ }^{0} \mathrm{C} / \mathrm{min}$, at flow of 100 $\mathrm{ml} / \mathrm{min}$ of pure $\mathrm{N}_{2}[26]$.

Afterwards, the DRIFTS spectra were gathered using a Thermo Scientific Nicolet iS10 FTIR spectrometer. A background, with pure $\mathrm{KBr}$, provided by Nicolet was always accumulated previous to sample collection.

After that, the room temperature Mössbauer spectroscopy (MS) measurements were carried out with a SEECo supplied spectrometer operating at constant acceleration mode with a $50 \mathrm{mCi}^{57} \mathrm{Co}$ gamma-ray source at $\mathrm{Rh}$ matrix made by Rietverc $\mathrm{GmbH}$.. The 1024 point raw data were analysed using a least square fitting spectral analysis program (PeakFit, Seasolve Software Inc., Framingham, Massachusetts).

Additionally, the magnetization curve ( $\mathrm{M}$ versus $\mathrm{H}$ ) was collected at room temperature (300 K) in the vibrating sample magnetometer (VSM), Lakeshore 7400 Series. The maximum magnetic field used was $2.2 \mathrm{~T}$.

Finally, to measure the BET (Brunauer-Emmett-Teller) specific surface area $\left(\mathrm{S}_{\mathrm{BET}}\right)[32]$ and the equivalent spherical particle diameter [33], the adsorption isotherms of nitrogen (Praxair, 99.99 \%) at $77 \mathrm{~K}$ for the akaganéite phase degassed at $423 \mathrm{~K}$ for 4 hours in high vacuum was obtained. As well, carbon dioxide (Praxair, $99.99 \%$ ) adsorption at temperatures of $300 \mathrm{~K}$ and $318 \mathrm{~K}$ and pressures up to 30 bar, i.e., the high pressure range, was investigated, in a Quantachrome iSorbHP-100 on samples degassed at $423 \mathrm{~K}$ for four hours in high vacuum $\left(10^{-6}\right.$ Torr $)$. The backfilling process was performed using helium (Praxair, 99.99\%). 


\section{Results and discussion}

\subsection{Characterization}

At first, in Fig. 1 are exhibited the XRD profiles of the AK-AS, AK-W and AK-Li. The XRD analysis revealed the presence of akaganéite and sodium chloride in the AK-AS material (Fig. 1a), while in the AK-W (Fig. 1b) and the AK-Li (Fig. 1c) only akaganéite was found. The XRD profiles of the AK-W and AK-Li materials were refined using the Pawley profile fitting method assuming the $I 2 / m$ space group [13]. Our results are exhibited in Table 1 together with data previously reported in literature for two different akaganéites $[9,13]$. The direct comparison of both sets of data yielded a slight dissimilarity in the parameters, possibly due to a different distribution of the chloride cations.

After that, in Fig. 2 are reported the SEM images corresponding to akaganéite, aggregates (Fig. 2a), particles (Fig. 2b) and crystallites (Fig. 2c) contained in the AK-W sample. The aggregates are compounds of ca. $30 \times 50 \mu \mathrm{m}^{2}$ formed by smaller particles of $10-50 \mu \mathrm{m}$ composed of spindle-shaped crystallites of $6 \times 10 \mu \mathrm{m}^{2}$ in parallel association [15]. EDX tests in five different spots per sample were performed. In Table 2 are shown the results, in which, since Li can't be analysed by EDX, so the reported figure was estimated using the amount of chloride. In addition, this analysis demonstrated that the washed akaganéite phase composition satisfy the $O / F e$ relation stoichiometry [10]; while, the amount of chloride is within an appropriate range [11]. Next, TGA analysis was performed. The profiles corresponding to the AK-W and AK-Li samples are reported in Figs. 3a and $3 \mathrm{~b}$ respectively. The effect of heating in akaganéite produce initially water removal up to $380 \mathrm{~K}\left(107{ }^{\circ} \mathrm{C}\right)$, later, from $443 \mathrm{~K}$ to $493 \mathrm{~K}\left(170-220{ }^{\circ} \mathrm{C}\right)$ 
dehydroxilation and finally the release of $\mathrm{HCl}$ at ca. $543 \mathrm{~K}\left(270{ }^{\circ} \mathrm{C}\right)[9,12]$; hence as is evidenced in both profiles the synthesized akaganéite behaved as expected.

In Fig. 4 are reported the DRIFTS spectra in the $500-1900 \mathrm{~cm}^{-1}$ (Fig. 4a) and $1800-3800 \mathrm{~cm}^{-1}$ regions of the AK-AS sample. In Fig $4 \mathrm{a}$ are observed peaks at $710 \mathrm{~cm}^{-1}$ and $850 \mathrm{~cm}^{-1}$ linked to the Fe-O stretching and O-H bend [34]. In Fig. $4 \mathrm{~b}$ the bands located on $1440 \mathrm{~cm}^{-1}$ and $1530 \mathrm{~cm}^{-1}$ are linked to the $\mathrm{C}-\mathrm{O}$ symmetric and asymmetric stretching vibrations distinctive of carboxylic acids [35] i.e., sodium citrate produced during the synthesis process. Further, a band at 2800$3400 \mathrm{~cm}^{-1}$, resulting from the interacting $\mathrm{OH}$ groups, while free $\mathrm{OH}$ groups exhibited a band at, $3400-3600 \mathrm{~cm}^{-1}[22]$.

\subsection{Magnetic behaviour}

In Fig. 5 are shown the magnetization curves of the AK-W (Fig. 5a) and AK-Li (Fig. 5b) samples collected at $300 \mathrm{~K}$. Bulk akaganéite shows an anti-ferromagnetic behaviour in which two spin sub-lattices are coupled along the [010] direction of the framework [36, 37]. This conduct is caused by the imbalance of the surface spins, effect that confer a net magnetic moment to the akaganéite nanoparticles [38,39. Even though, in akaganéite is assumed an anomalous antiferromagnetic behaviour [40-42]; since iron is located in four non-equivalent sites near to the chlorine anion sites; hence, the chlorine content has an altering effect in the magnetic performance of this material [43]. Additionally, this antiferromagnetic behaviour could be masked, since the crystallite sizes are very small, then, the $\mathrm{Fe}^{3+}$, ions experiences an averaged to zero effective internal magnetic field producing a super-paramagnetic behaviour [45], as was observed in Fig. 5 for both materials. 
The Mössbauer spectra of the AK-W and AK-Li phases, collected at $300 \mathrm{~K}$ are reported in Fig 6a and Fig. 6b. Both spectra were fitted with two overlapping paramagnetic doublets yielding similar values for the measured parameters. Our results are shown in Table 3 at the same time as data previously reported in literature for two different akaganéites [34,44] to make a direct comparison with our results. Hence was found that the data obtained by us is very similar to previously reported Mössbauer parameters measured for akaganéite phases [45]. On this ground, we concluded that the presence of lithium in the framework did not influence the environment of the octahedral, $\mathrm{Fe}^{3+}$, sites, since it is a very small ion [46]. Moreover, the fact that both spectra were fitted with two overlapping paramagnetic doublets indicates the presence of two sites for iron in the framework. Additionally was observed a super-paramagnetic behaviour for both tested materials.

\subsection{Framework representation.}

The gathered XRD, EDX and DRIFTS data made possible conclude that the composition in weight per-cent (wt. \%) of the AK-As sample was, akaganéite (48 wt\%), sodium chloride (wt. 47 $\%$ ) and sodium citrate (wt, $5 \%$ ). Meanwhile, using the crystallographic information reported for

the AK-WAK-Li phases (Table 1), the Mössbauer spectra (Fig. 5), together with the atomic positions and occupancy factors reported by Post et al [13] and the Wyckoff sites taken from the International Tables for Crystallography [47], was produced a representation (Fig. 7) of the akaganéite framework along the [010] crystallographic direction with the Bruker DIFFRACplus TOPAS ${ }^{\mathrm{TM}}$ software; where are evidenced $4 \times 4 \AA^{2}$ wide channels, in which the chloride ions are stabilized by hydrogen bonding from bulk $\mathrm{OH}$ groups and are reported the cell coordinate along the [010] direction for both Fe ions. 


\subsection{Adsorption study}

\subsubsection{Isotherm collection}

To gather the adsorption data to get the isotherms, the total adsorption amount was measured with the volumetric method, in the "low" (up to 7 Bar for carbon dioxide and up to 1 bar for nitrogen) and "high" pressure (up to 30 Bar for carbon dioxide) ranges, using the following equation [48], $n_{a}=n_{t}-\rho_{g} V_{g}$, in which, $n_{a}$, is the net amount adsorbed, $n_{t}$, is the total amount

adsorbed, $\rho_{g}$, the adsorbate density and, $V_{g}$, the dead volume, which include the pore volume of the adsorbent. To measure, $V_{g}$, was used a reference gas, specifically, He, for which the amount adsorbed, $n_{a}$, is considered zero i.e., $n_{a}=n_{t}-\rho_{g} V_{g}=0$, hence, $V_{g}=n_{t} / \rho_{g}$, where, $\rho_{g}$, is the density of He [49].

\subsubsection{Low pressure carbon dioxide and nitrogen adsorption}

To characterize the microporous adsorption space, the low pressure carbon dioxide at $300 \mathrm{~K}$ adsorption on the AK-AS and the AK-Li materials data, Fig 8a and Fig. 8b respectively, were fitted to the linear form of the Dubinin-Radushkevitch (D-R) adsorption isotherm equation [50]:

$$
\ln \left(n_{a}\right)=\ln \left(N_{a}^{0}\right)-\left(\frac{R T}{E}\right)^{2} \ln \left(\frac{P_{0}}{P}\right)^{2}
$$

Wherein, $n_{a}$, is the amount adsorbed, $\left(n_{a} \approx n_{t}\right.$, in the low pressure range); $P / P_{0}$, the relative pressure, $E$, the characteristic energy of adsorption, and, $N_{a}^{0}$, the maximum amount adsorbed in the micropore. The fitting process made possible the calculation of, $E$, and, $N_{a}^{0}$, while $W_{0}$, the micropore volume, was computed using the Gurtvich rule, $W_{0}=N_{a}^{0} V_{C_{2}}$, where the carbon 
dioxide molar volume, $V_{\mathrm{CO}_{2}}=48.3 \mathrm{~cm}^{3} / \mathrm{mol}$; whereas, $q_{\text {iso }}$, the isosteric heat of adsorption was calculated using the following equation, $q_{\text {iso }}(\theta=0.37)=1.16 E($ Table 4$)[22,51]$.

To describe the akaganéite outer surface the collected nitrogen at $77 \mathrm{~K}$ in the low pressure range adsorption data were fitted (Fig. 8c) to the Brunnauer-Emmet-Teller (BET) isotherm equation in linear form [52]:

$$
y=\frac{x}{n_{a}(1-x)}=\left(\frac{1}{N_{m} C}\right)+\left(\frac{C-1}{C N_{m}}\right) x=b+m x
$$

Where: $b=\left(1 / N_{m} C\right), m=\left(C-1 / N_{m} C\right), y=x / n_{a}(1-x), n_{a}$, is the amount adsorbed, $N_{m}$, is the monolayer capacity, $C=C(T), x=P / P_{0} \quad(0.05<x<0.4)$. On this ground, $N_{m}$ was calculated yielding, $N_{m}=2.16 \mathrm{mmol} / \mathrm{g}$, then the surface area was calculated as follows: $S=N_{m} N_{A} \sigma$, in which, $N_{A}$, is the Avogado number, and $\sigma\left(N_{2}\right)$, the nitrogen molecule cross-sectional area $\left(\sigma\left(N_{2}\right)=\right.$ $0.162 \mathrm{~nm}^{2}$ for $N_{2}$ at $77 \mathrm{~K}$ ) giving up, $S=220 \mathrm{~m}^{2} / \mathrm{g}$.

Hence, the carbon dioxide molecule, freely passed through the tested material pores; given that, the collected $\mathrm{CO}_{2}$ adsorption data was exactly fitted by the DR isotherm equation; while, nitrogen was only adsorbed on the outer surface; since the gathered $\mathrm{N}_{2}$ adsorption data was accurately fitted by the BET isotherm equation $[5,25]$. Thereafter, since the diameters of the carbon dioxide and nitrogen molecules are $\sigma_{\mathrm{CO}_{2}}=3.30 \AA$ and $\sigma_{\mathrm{N}_{2}}=3.64 \AA$ respectively [53], we concluded that the tunnels existing in the akaganéite framework [3] have a pore diameter, $d \approx 3.5 \pm 0.1 \AA$.

Finally, the equivalent particle diameter of the synthesized akaganéite, was measured using the BET specific surface area $\left(\mathrm{S}_{\mathrm{BET}}\right)$ by means of the equation, $\Phi_{\mathrm{BET}}=6 /\left(\mathrm{S}_{\mathrm{BET}}\right) \rho$, where $\rho$ is the akaganéite density [32]. Then, the BET equivalent spherical diameter $\left(\Phi_{\mathrm{BET}}\right)$, for, $\rho=3.5 \mathrm{~g} / \mathrm{cm}^{3}$, 
yielded, $\Phi_{\mathrm{BET}}=8$ (1) nm, a value, within the experimental error, equivalent to the Gaussian spherical diameter measured with the XRD data $\left(\Phi_{\mathrm{G}}=10(1) \mathrm{nm}\right)[1,9,13]$.

\subsubsection{Carbon dioxide adsorption heat}

The collected up to $9 \mathrm{Bar}$, at $273 \mathrm{~K}$ and $300 \mathrm{~K}$, carbon dioxide adsorption data were used to calculate the isosteric heat of adsorption utilizing the Clausius-Clapeyron equation [54]:

$$
q_{i s o} \approx R T^{2}\left[\frac{d \ln P}{d T}\right]_{n_{a}} \approx R T_{1} T_{2}\left(\frac{\ln P_{2}-\ln P_{1}}{T_{2}-T_{1}}\right)_{n_{a}}
$$

Where, $n_{a}$, is the amount adsorbed in $\mathrm{mmol} / \mathrm{g}$, since $n_{a} \approx n_{t}$ in the pressure range of interest, $T_{i}$, is temperature and $P_{i,}$, equilibrium adsorbate pressure at constant loading. In Figs. 9a and 9b are reported the isosteric heat of adsorption, calculated according to equation " 3 " for the AK-W and the AK-Li phases versus coverage $\left(\theta=n_{a} / N_{a}^{0}\right)$. These data fairly well coincide with the isosteric heat of adsorption calculated using the D-R adsorption isotherm equation (Table 4). Next, we know that when a molecule is adsorbed, it is subjected to the following interactions: dispersion, $\varphi_{\mathrm{D}}$, repulsion, $\varphi_{\mathrm{R}}$, polarization, $\varphi_{\mathrm{P}}$, field dipole, $\varphi_{\mathrm{E} \mu}$, field gradient quadrupole, $\varphi_{\mathrm{EQ}}$, adsorbate-adsorbate interaction, $\varphi_{\mathrm{AA}}$ and in some cases the acid-base interaction, $\varphi_{\mathrm{AB}}[55,56]$. Subsequently, since carbon dioxide is a molecule showing a noticeable quadrupolar moment $\left(Q_{\mathrm{CO} 2}=-4.3 \times 10^{-42} \mathrm{Cm}^{2}[57]\right)$ and slight acidity [58]; hence, it should strongly interact within the akaganéite micropores. The strong interaction at low coverage is to a large extent a consequence of the small diameter of the approximately cylindrical [59] akaganéite pores and the specific interaction of the carbon dioxide molecule with the $\mathrm{Cl}^{-}$anions [59]. Incidentally, when carbon dioxide interacts with another chemical species in some cases the electron transfer is from 
the chemical species to the $\mathrm{CO}_{2}$ molecule, producing a, $\mathrm{CO}_{2}^{-}$, anion conversely when the negative charge transfer is from the molecule to the chemical species a $\mathrm{CO}_{2}^{+}$cation is generated [61]. Consequently, when a $\mathrm{CO}_{2}$ molecule, interact with a negatively charged $\mathrm{Cl}^{-}$anion, owing to the electronegativity of halogens the electron transfer is from the $\mathrm{CO}_{2}$ molecule to the $\mathrm{Cl}^{-}$anion, giving rise to a $\mathrm{CO}_{2}^{+}$cation attractively interacting with the $\mathrm{Cl}^{-}$anion, producing the specific interaction of the carbon dioxide molecule with the $\mathrm{Cl}^{-}$anions $[62,63]$.

\subsubsection{High pressure carbon dioxide adsorption}

In Fig. 10 are exhibited the high pressure carbon dioxide adsorption isotherms on the AK-W (Fig. 10a) and the AK-Li (Fig. 10b) phases at different temperatures; while in Fig. 10c are compared the adsorption in both materials at $\mathrm{T}=300 \mathrm{~K}$. These isotherms shows that the amount adsorbed continuously increased for both materials, i.e. the adsorption process was not saturated when the pore volume of the adsorbent was filled by the adsorbent. This fact is only possible when the adsorbent for the course of the adsorption processes experience deformation, explicitly, expansion of the adsorbent framework [63-66].

To Thermodynamically work out the effect of the high pressure adsorption process on the akaganéite, the adsorbent plus the adsorbed phase was considered as a solid solution, i.e., the adsorbate-adsorbent system $(a A)$, where, the internal energy per unit mass of adsorbent can be calculated as follows [51]:

$$
\bar{U}_{a A}=T \bar{S}_{a A}-P \bar{V}_{a A}+\mu_{a} n_{a}+\mu_{A}
$$

Where, $U_{a A}, S_{a A}$ and $V_{a A}$ are the internal energy, entropy and volume of the system $a A$, while $\mu_{a}$ ( in $\left.J / m o l\right)$ and $\mu_{A}$ (in $J / K g$ ) are the chemical potentials of the adsorbed and solid phases in 
the solution and, $n^{a}$, and $m$ the amount of moles of adsorbate and the adsorbent mass for the system $a A$ : meanwhile, $\bar{U}_{a A}=U_{a A} / m, \bar{S}_{a A}=S_{a A} / m, \bar{V}_{a A}=V_{a A} / m$ and $n^{0}=n_{a} / m$ [67].

In addition, the internal energy for the unit mass of empty adsorbent is given by:

$$
\bar{U}_{A}=T \bar{S}_{A}-P \bar{V}_{A}+\mu_{A}^{0}
$$

Thereafter [68]:

$$
U_{a}=\bar{U}_{a A}-\bar{U}_{A}=T S_{a}-P V_{a}+\mu_{a} n_{a}+\Phi,
$$

Where, $S_{a}=\bar{S}_{a A}-\bar{S}_{A}, V_{a}=\bar{V}_{a A}-\bar{V}_{A}$ and $\Phi=\mu_{A}-\mu_{A}^{0}$.

Now, since the Grand potential is defined as: $\Omega=U-T S-\mu n$,

Then:

$$
\Omega_{a}=U_{a}-T S_{a}-\mu_{a} n_{a}-\Phi
$$

And:

$$
d \Omega_{a}=d U_{a}-T d S_{a}-S_{a} d T-d \mu_{a} n_{a}-\mu_{a} d n-d \Phi
$$

Accordingly:

$$
d \Omega_{a}=-P d V_{a}-S_{a} d T-n_{a} d \mu_{a},
$$

But since, $d \mu_{a}=d \mu_{g}=R T \ln f$, where, $f$, is the fugacity of the adsorbate, hence [69]:

$$
d \Omega_{a}=-P d V_{a}-S_{a} d T-n_{a} R T \ln f=d \Phi .
$$

But since, $\Omega_{a}=\Phi$, accordingly, for $T=$ const:

$$
\Phi=R T \int_{0}^{f} n_{a} d(\ln f)-V_{a} P
$$

Furthermore, as was stated by Bering and Serpinskii within the frame of the osmotic theory of adsorption [70], $\Phi=\Pi W$, hence: 


$$
\Pi W=R T \int_{0}^{f} n_{a} d(\ln f)-V_{a} P
$$

Where, $W$, is the micropore volume of the adsorbent and, $\Pi=P^{0}-P$, is the osmotic pressure where $P^{0}$, is a pressure which can compress the adsorbate and produce the same effect created by an adsorption field, i.e., an amount, $n_{a}$, of adsorbed molecules [5]. Accordingly, in order to operate with equation "6", the fugacity of carbon dioxide must be calculated. In this regard, this parameter was computed by using the following expression [71]:

$$
\ln f=\ln P+\left[b-\frac{a}{R T}\right]\left(\frac{P}{R T}\right)
$$

In which, $a$ and $b$ are the van der Waals parameters for carbon dioxide, specifically, $a=36.58\left[\left(\mathrm{~m}^{3} / \mathrm{mol}\right)^{2} \mathrm{~Pa}\right]$ and $b=42.9\left[\mathrm{~m}^{3} / \mathrm{mol}\right]$. Accordingly, in Fig. 11 are exhibited the $\Pi$ versus $\mathrm{P}$ plot calculated using equation " 6 " and the adsorption data reported in Fig. 10c for the high pressure adsorption of carbon dioxide on both tested materials at $\mathrm{T}=300 \mathrm{~K}$. These diagrams (Fig. 11) shows a maximum for the osmotic pressure versus pressure plot, indicating an effect, recognized as a pressure-responsive framework opening sorption event [58-60]. Consequently this phenomenon took place due to a structural transformation induced in the framework by the pressure exercised by the adsorbate on the adsorbent [66]. This fact opens new applications for akaganéite as a high pressure adsorbent.

\section{Conclusions}

A mixture of akaganéite nanoparticles and sodium chloride was synthesized by a new route and then modified by washing and then by Li exchange. The key questions addressed in the reported research were the elucidation of the structure of the as-synthesized and Li exchanged akaganéite, 
together with the investigation of their adsorption space and framework expansion effect. The structural analysis was performed with: X-ray diffraction, thermo-gravimetric analysis, diffuse reflectance infrared Fourier transform spectrometry, Mössbauer spectrometry and magnetic measurements. As well, low pressure nitrogen and high pressure carbon dioxide adsorption experiments were performed. The gathered characterization information, related to the assynthesized mixture (AK-AS), the washed sample (AK-W) and the Li exchanged phase (AK-Li) made possible to conclude that the composition in weight per-cent (\%) of the mixture was akaganéite (48\%), sodium chloride (47\%) and sodium citrate (5\%). Additionally, the refining of the X-ray profile of the akaganéite phase made possible to conclude that it crystallized in a structure exhibiting the symmetry of the $I 2 / m$ space group. Meanwhile, the SEM images corresponding to the akaganéite phase particles exhibited diameters distributed between, 10 to 50 $\mu \mathrm{m}$ shaped by smaller elongated akaganéite crystals whose equivalent spherical diameter were 9 nm. Furthermore, the Mössbauer and magnetization information allowed us to conclude that $\mathrm{Fe}^{3+}$ ions are placed on two sites where their spin magnetic moments must be magnetically linked to produce an antiferromagnetic behaviour; however, the obtained akaganéite displayed a superparamagnetic behaviour. Meanwhile, the TGA data revealed that the produced akaganéite phases exhibited a standard performance under heating. Meanwhile, the low pressure nitrogen adsorption study of the pure akaganéite phase allowed the calculation of the specific surface area, yielding, $\mathrm{S}_{\mathrm{BET}}=220 \mathrm{~m}^{2} / \mathrm{g}$. Meanwhile the high pressure carbon dioxide adsorption data indicated that a pressure-responsive framework opening sorption event, took place due to a structural transformation for the adsorbent framework induced by the guest molecules. The obtained results open the possibility of new application for these materials not only as adsorbents but as well as sensors and precursors for catalysts. 


\section{Acknowledgements.}

The authors R.R.M., F.L., C.R.M. and R.P., acknowledges the financial support provided by the US Department of Energy through the Massey Chair project at the University of Turabo and recognize the support of the National Science Foundation under the project CHE-0959334. As well we acknowledge the help provided by Dr. Pedro Fierro in the collection of the magnetization curves in the Mayaguez Campus of the University of Puerto Rico. 


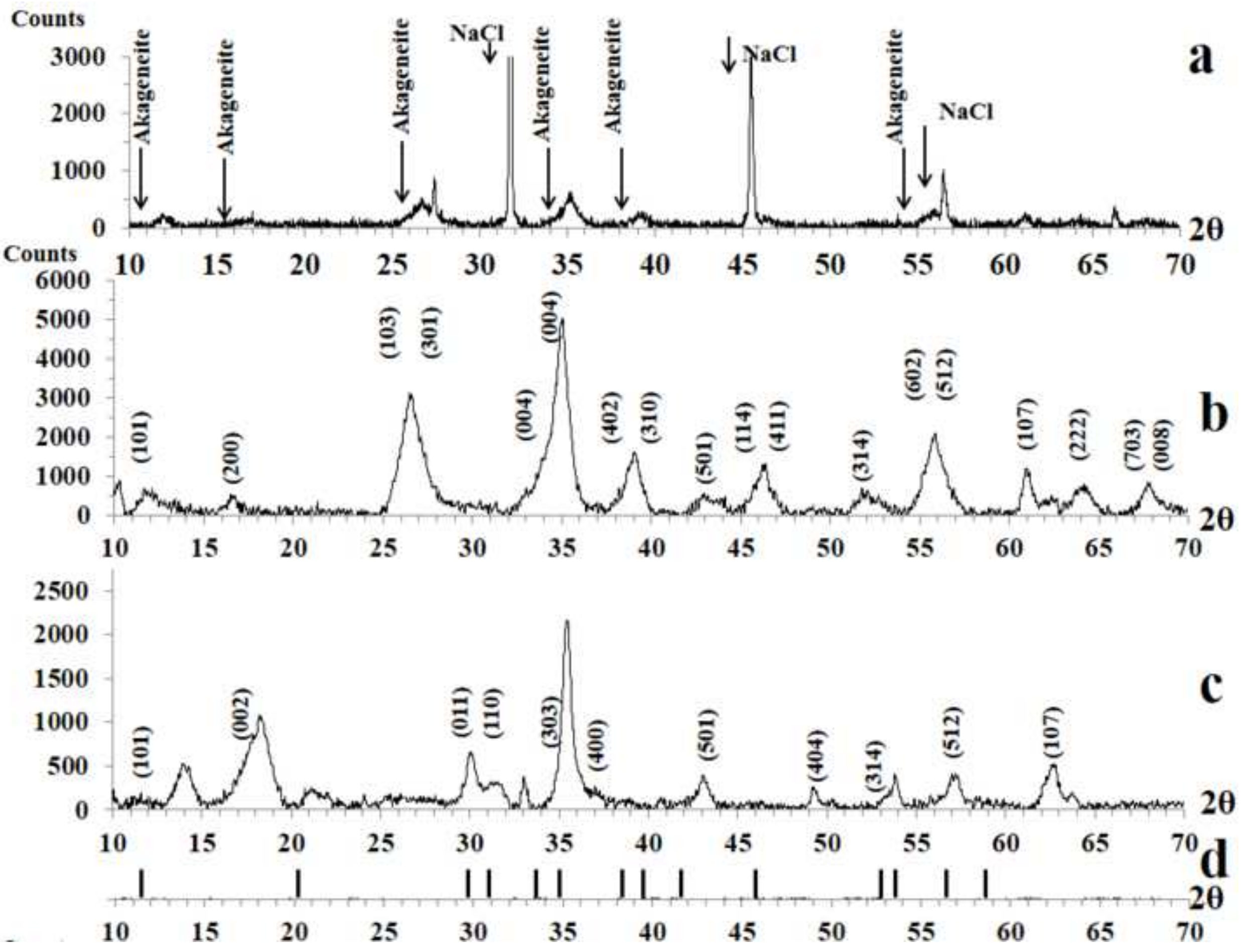



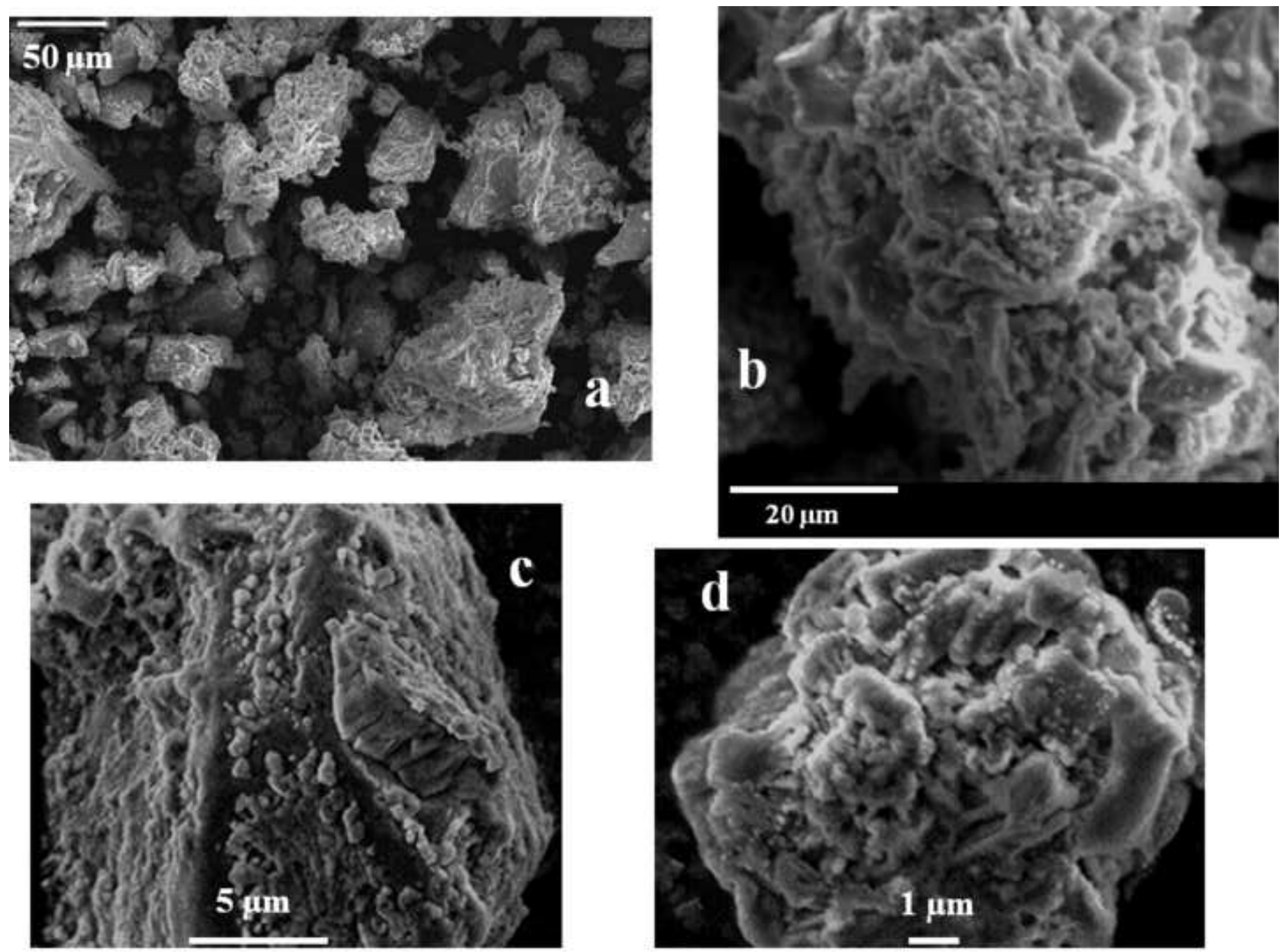

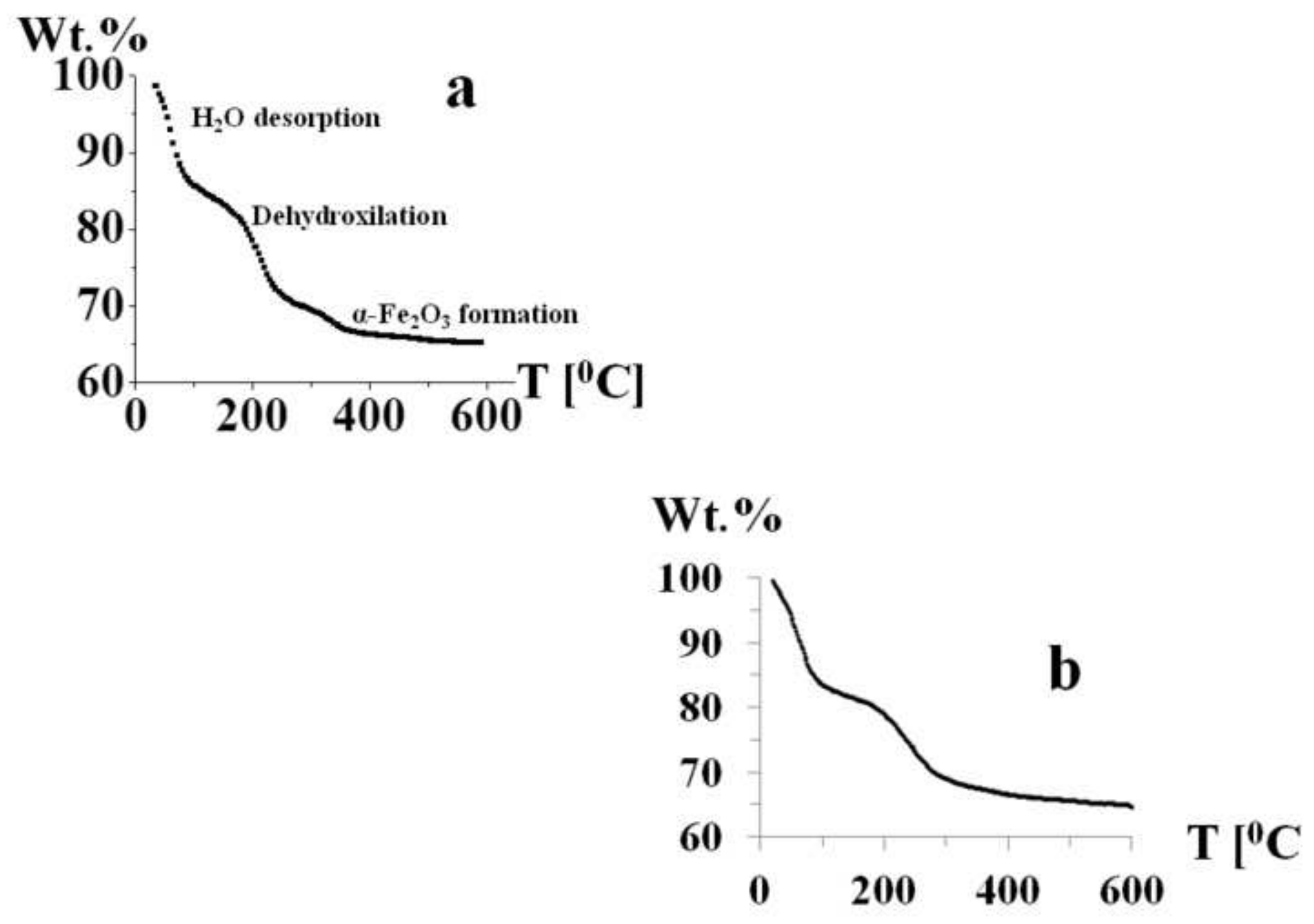


\section{Absorbance}

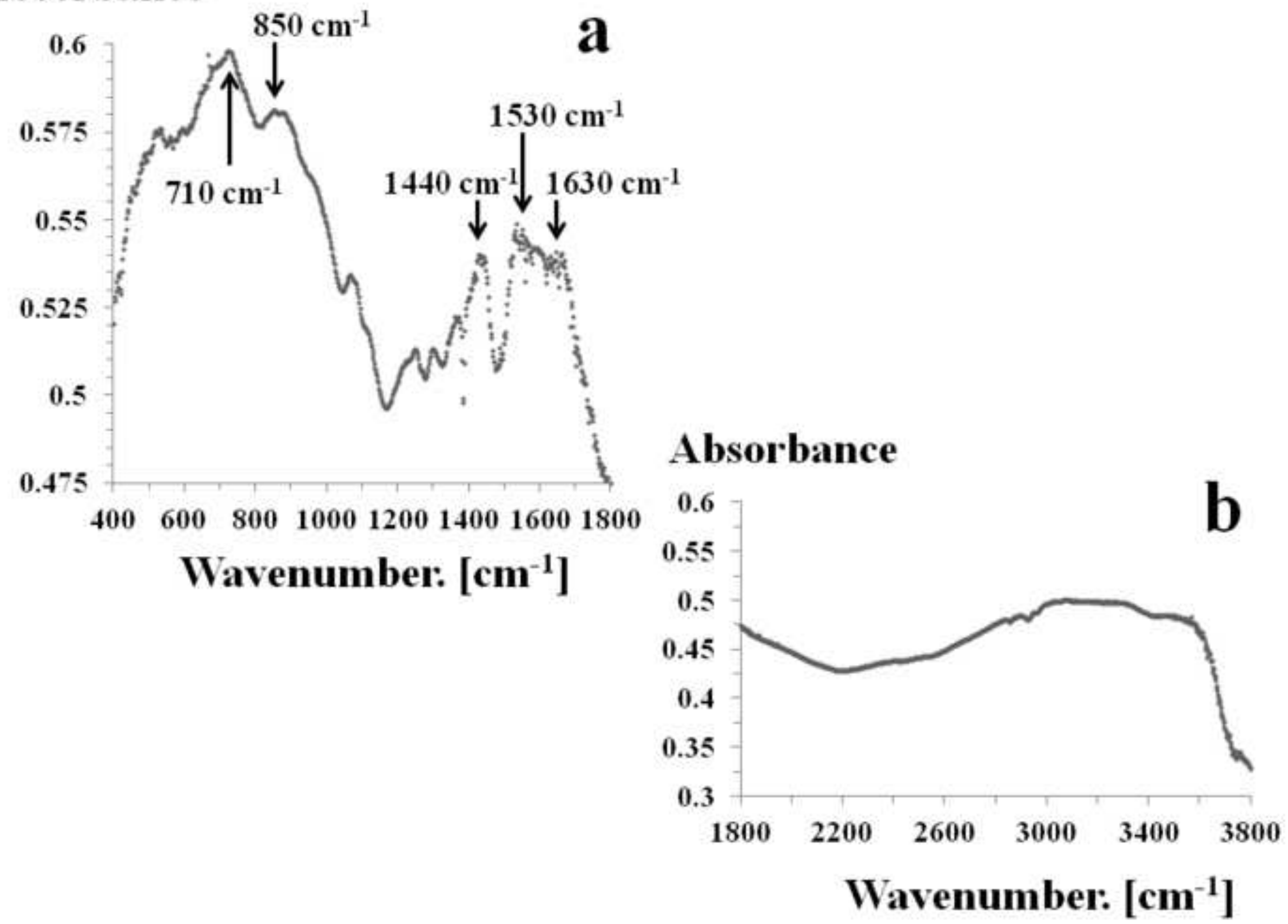


H

Fe

$\mathrm{O} O$
$\mathrm{CI}$

$+6$

$c$
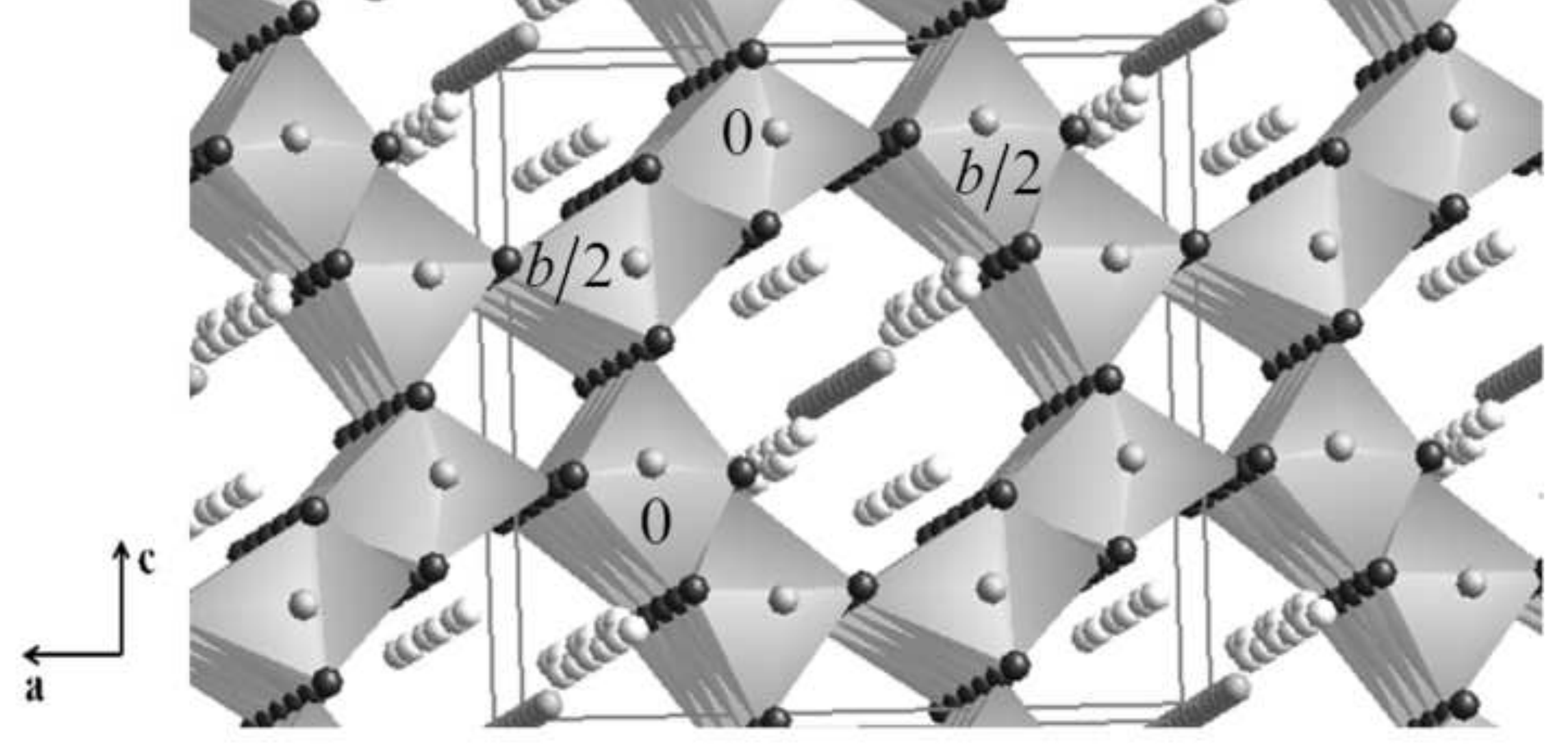
$M[\mathrm{emu} / \mathrm{g}]$

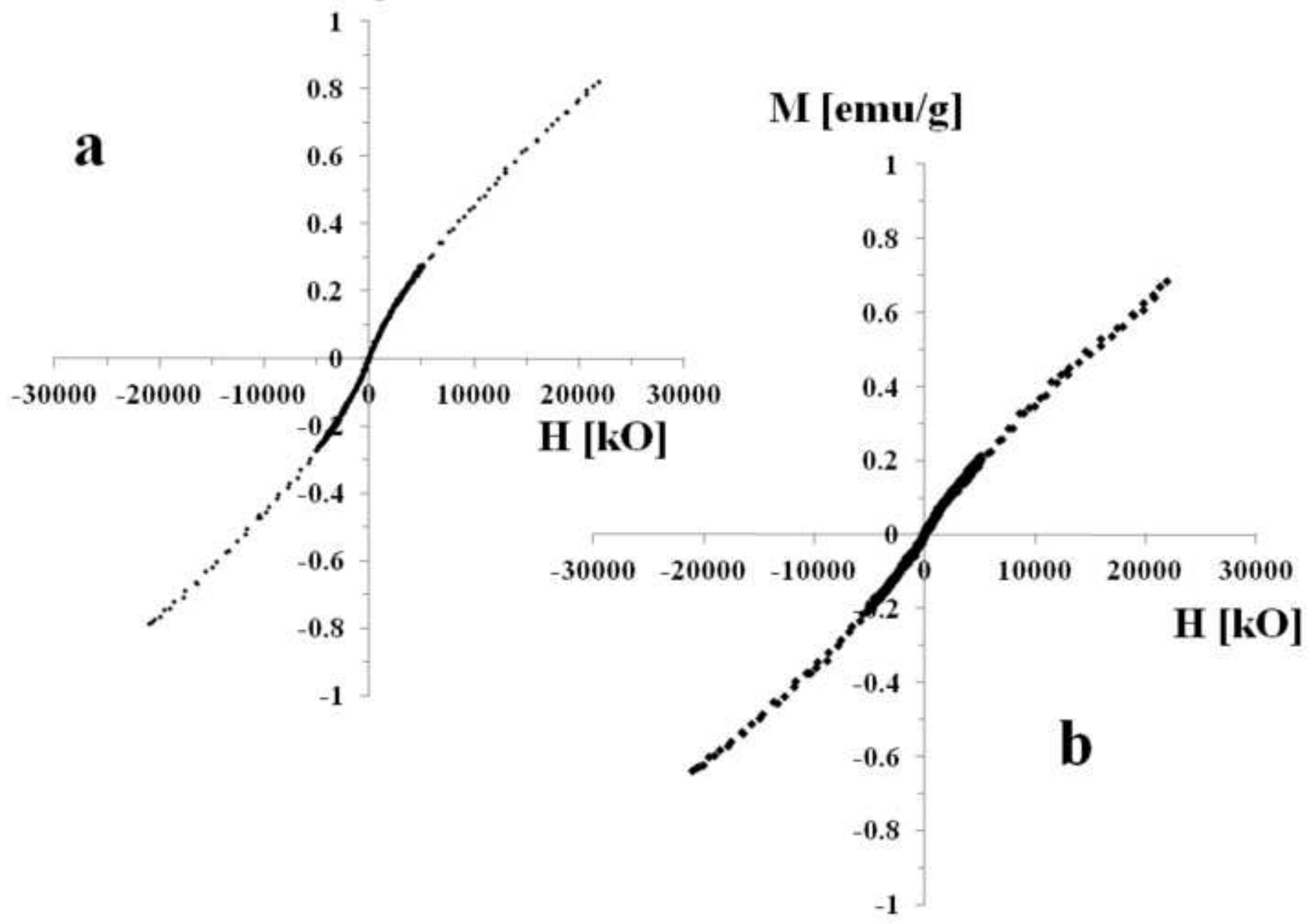


Absorption [\%]

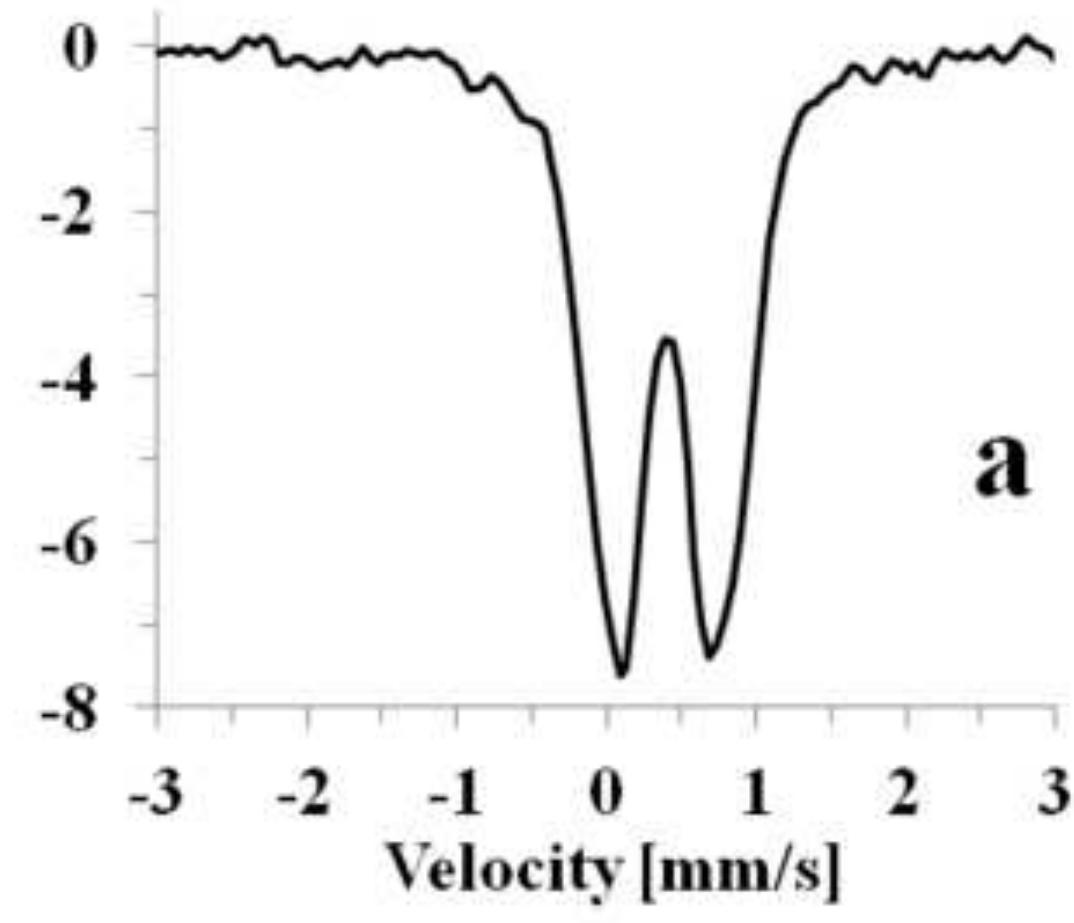

Absorption [\%]

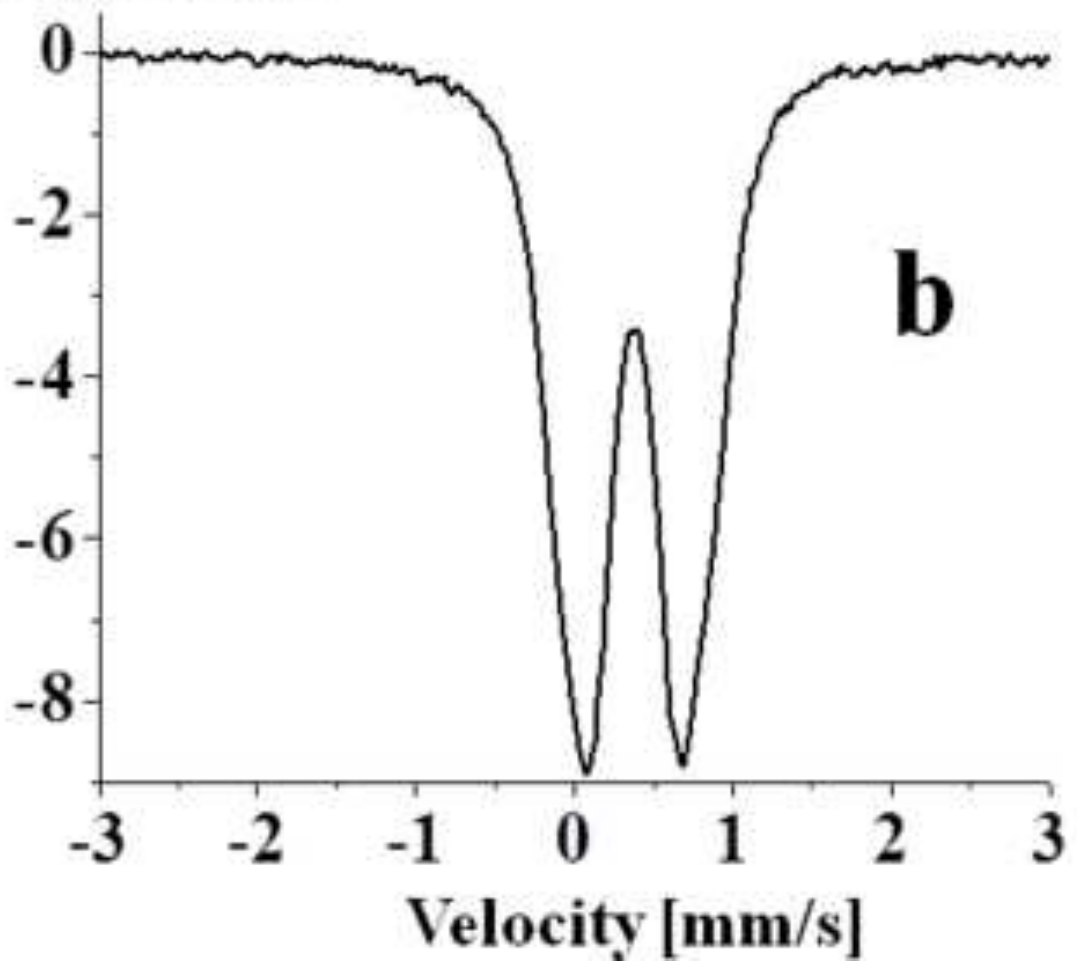



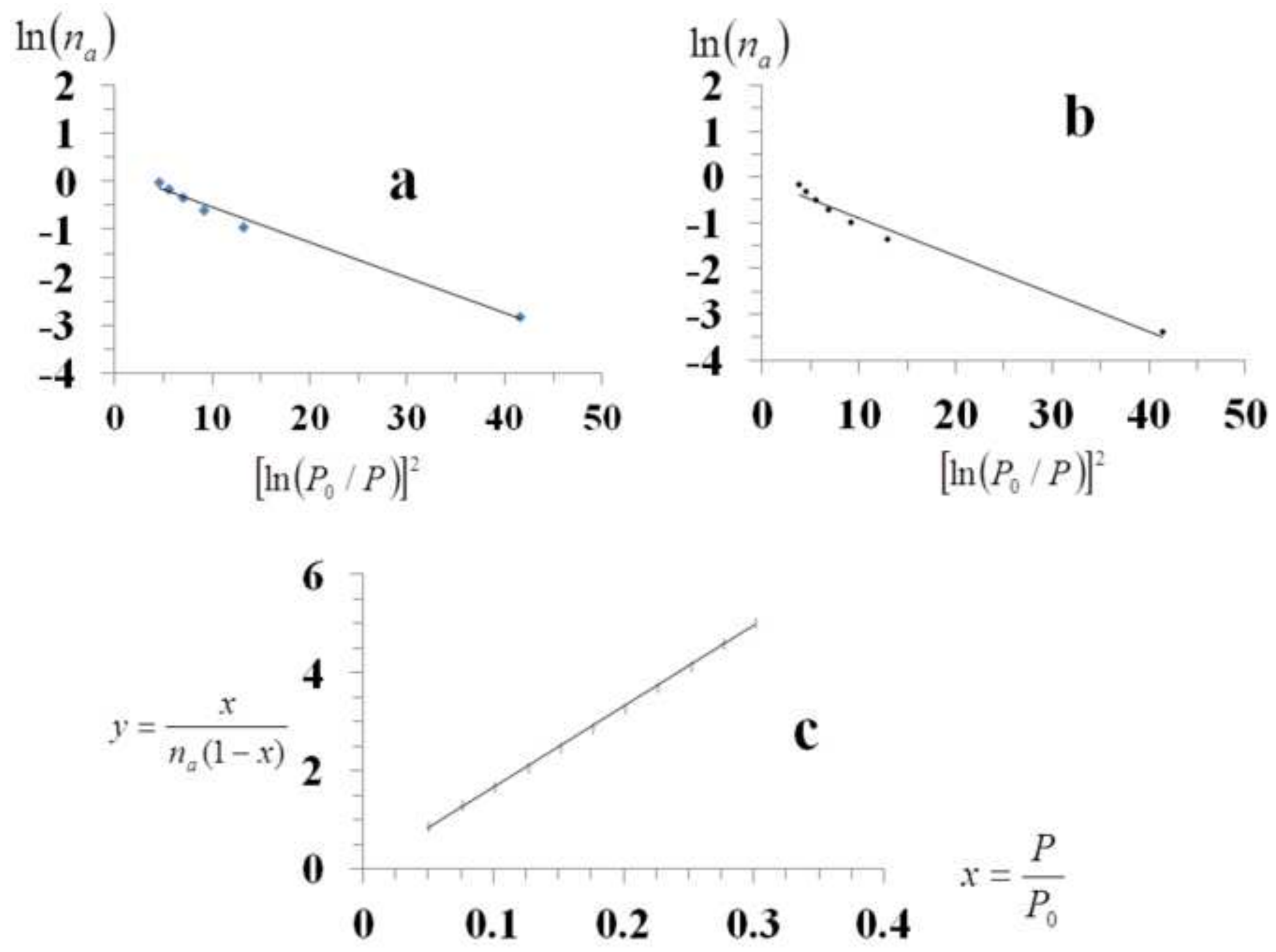
$q_{\text {iso }}[\mathrm{kJ} / \mathrm{mol}]$

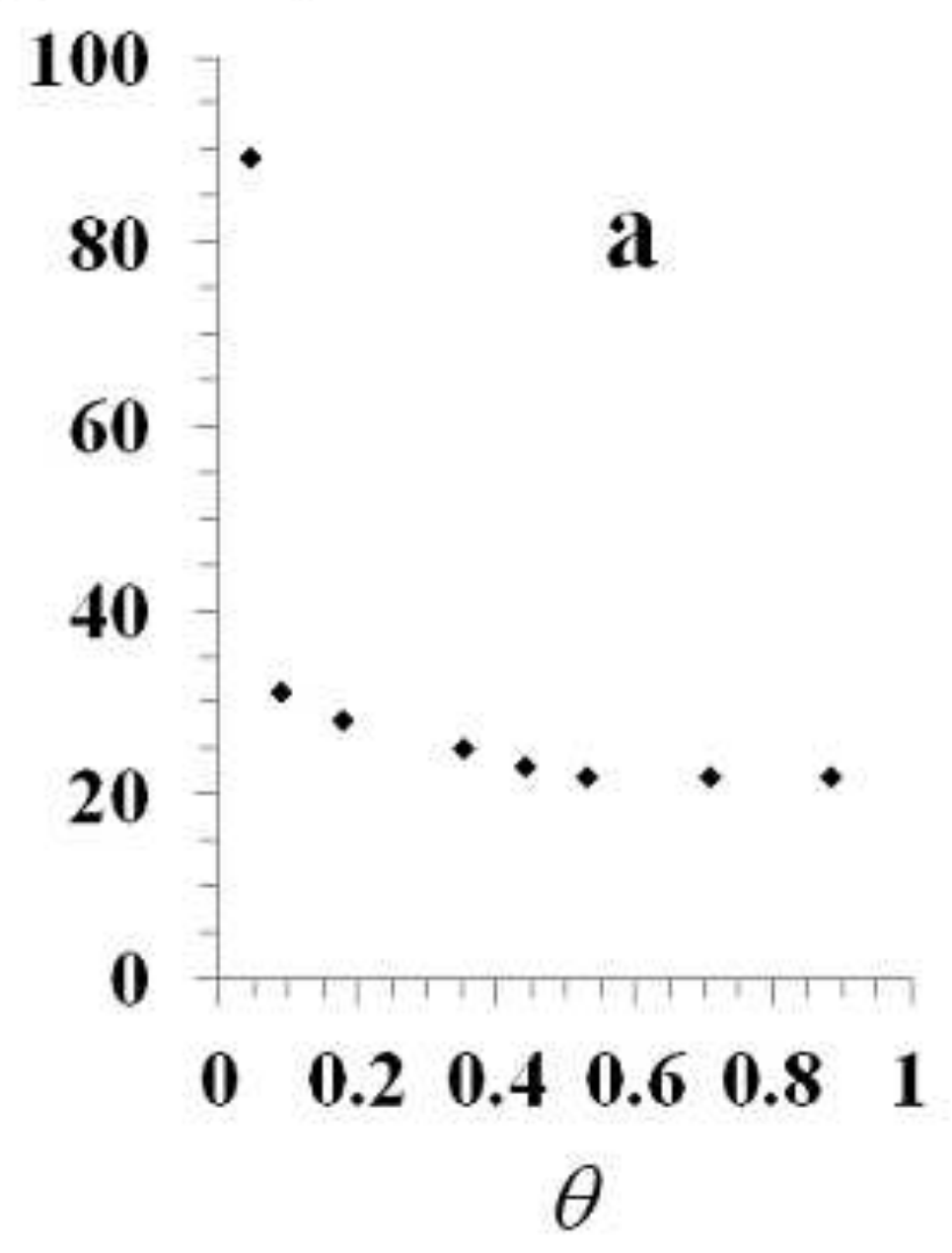

$q_{\text {iso }}[\mathrm{kJ} / \mathrm{mol}]$

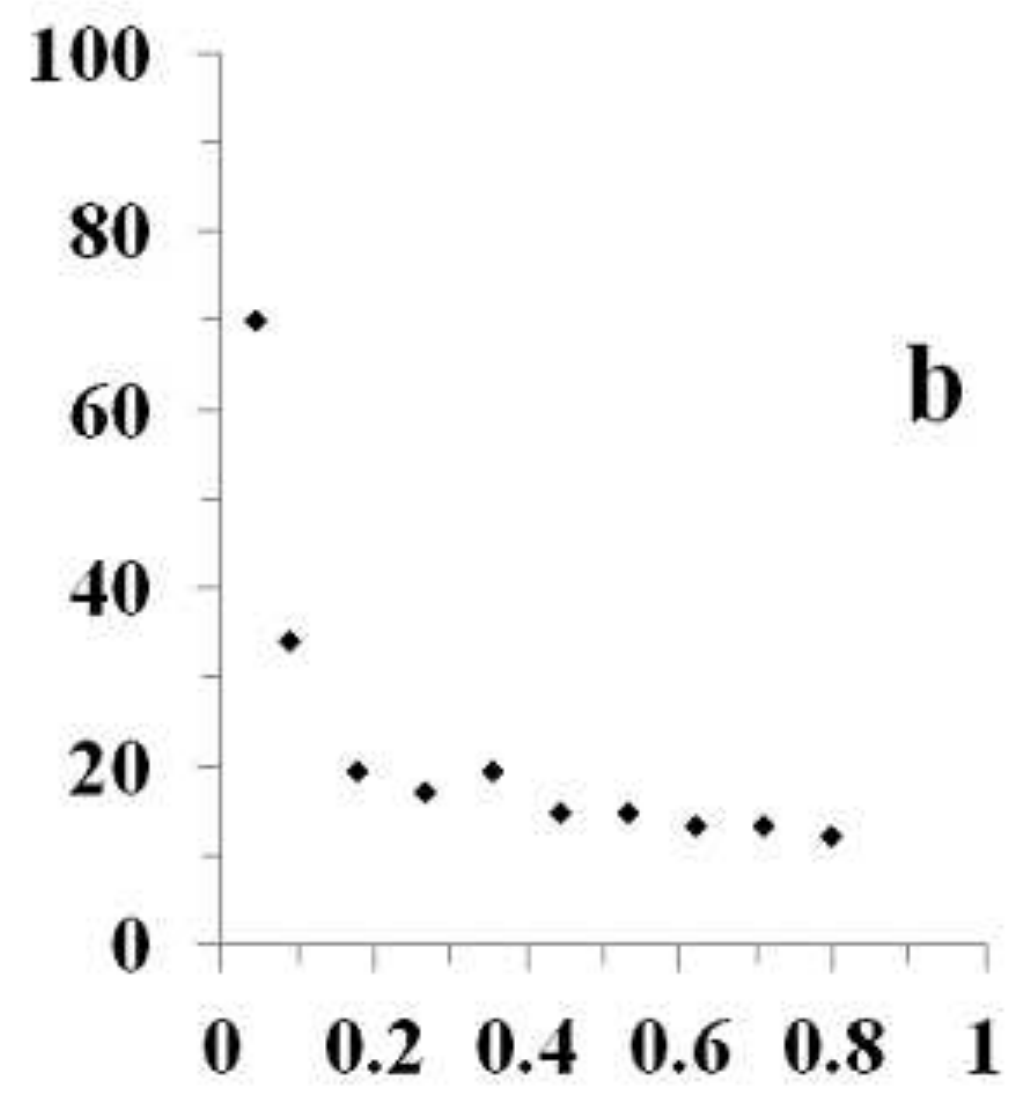

$\theta$ 


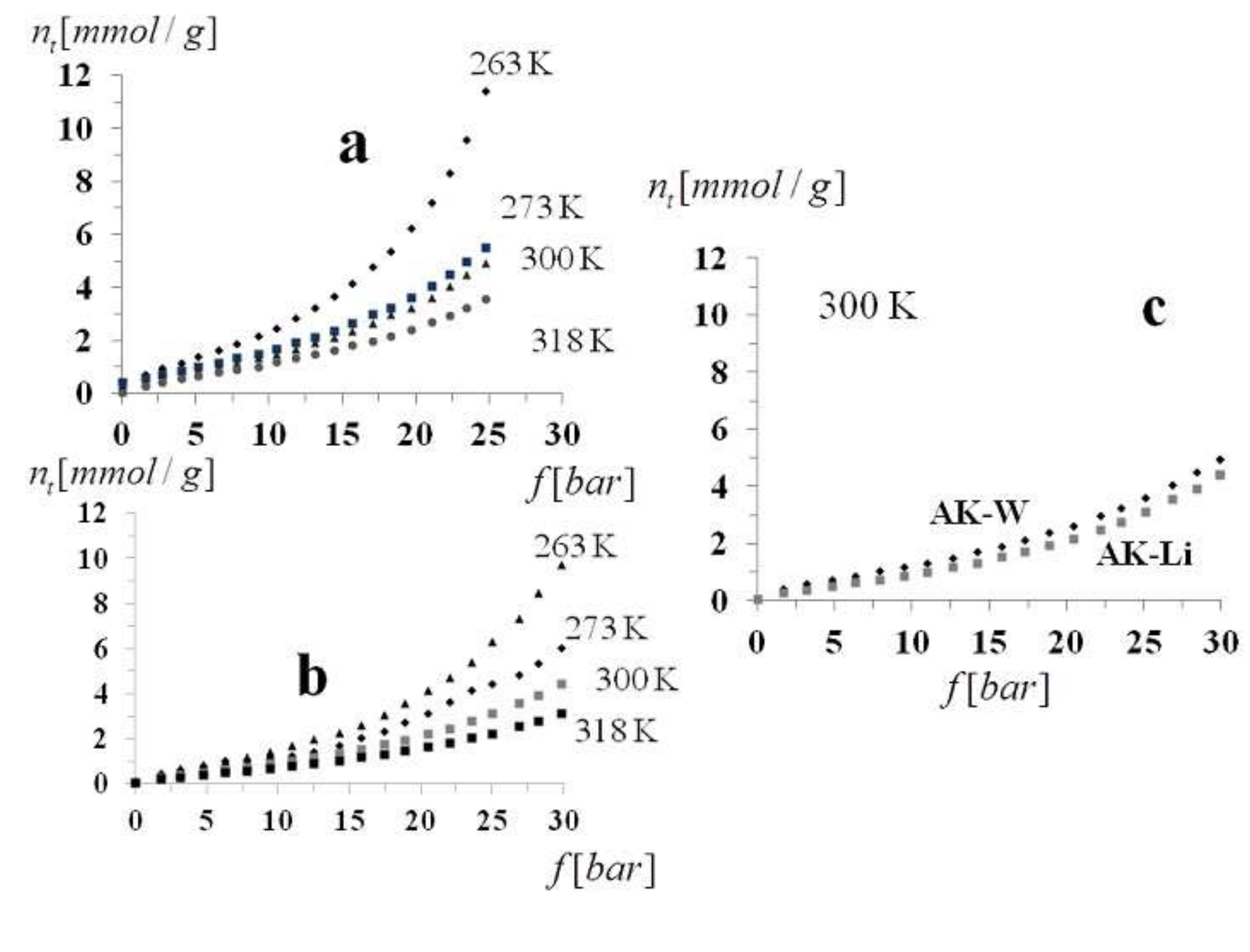

$n_{t}[\mathrm{mmol} / \mathrm{g}]$

. 


\section{$\Pi[M P a]$}

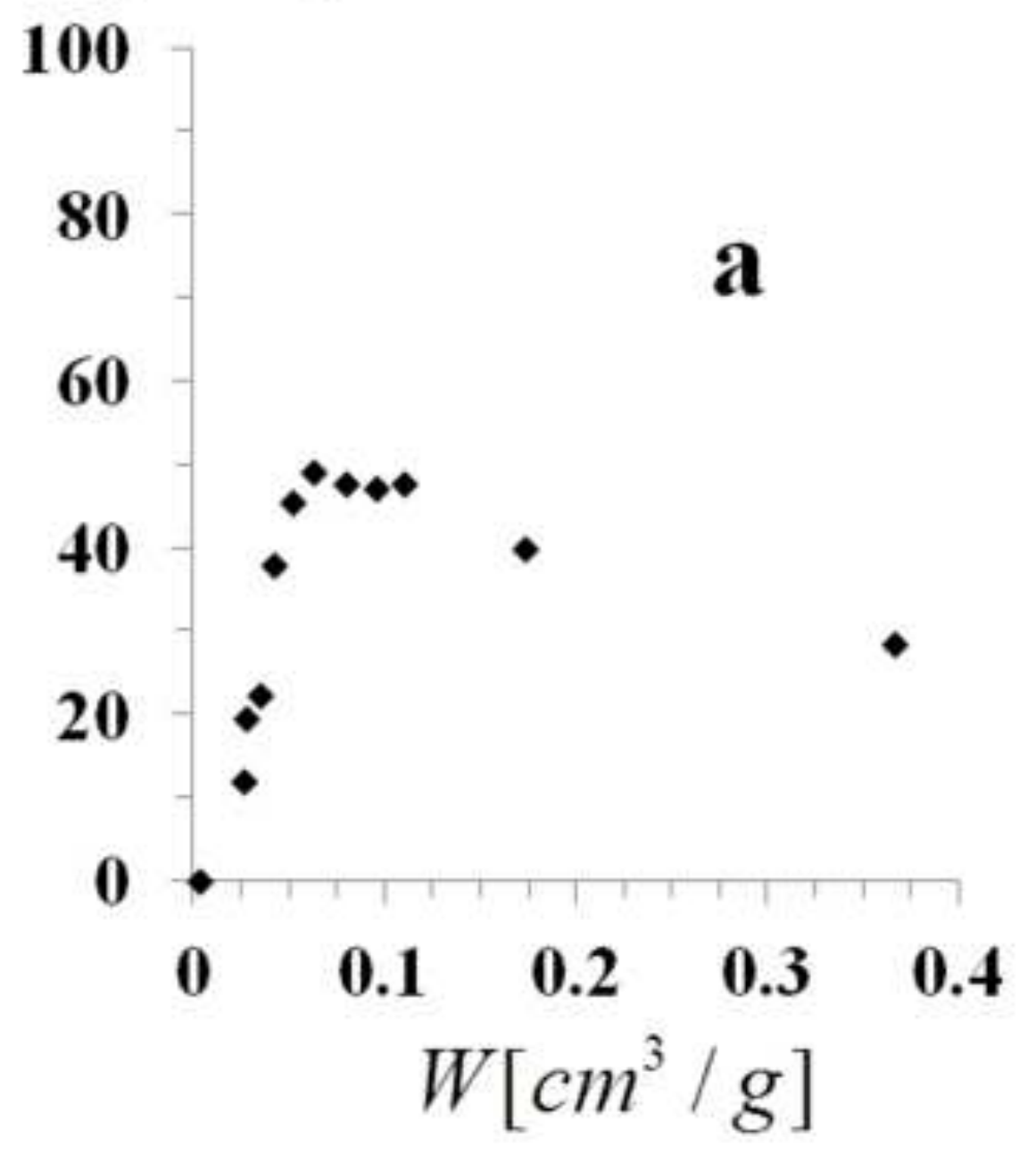

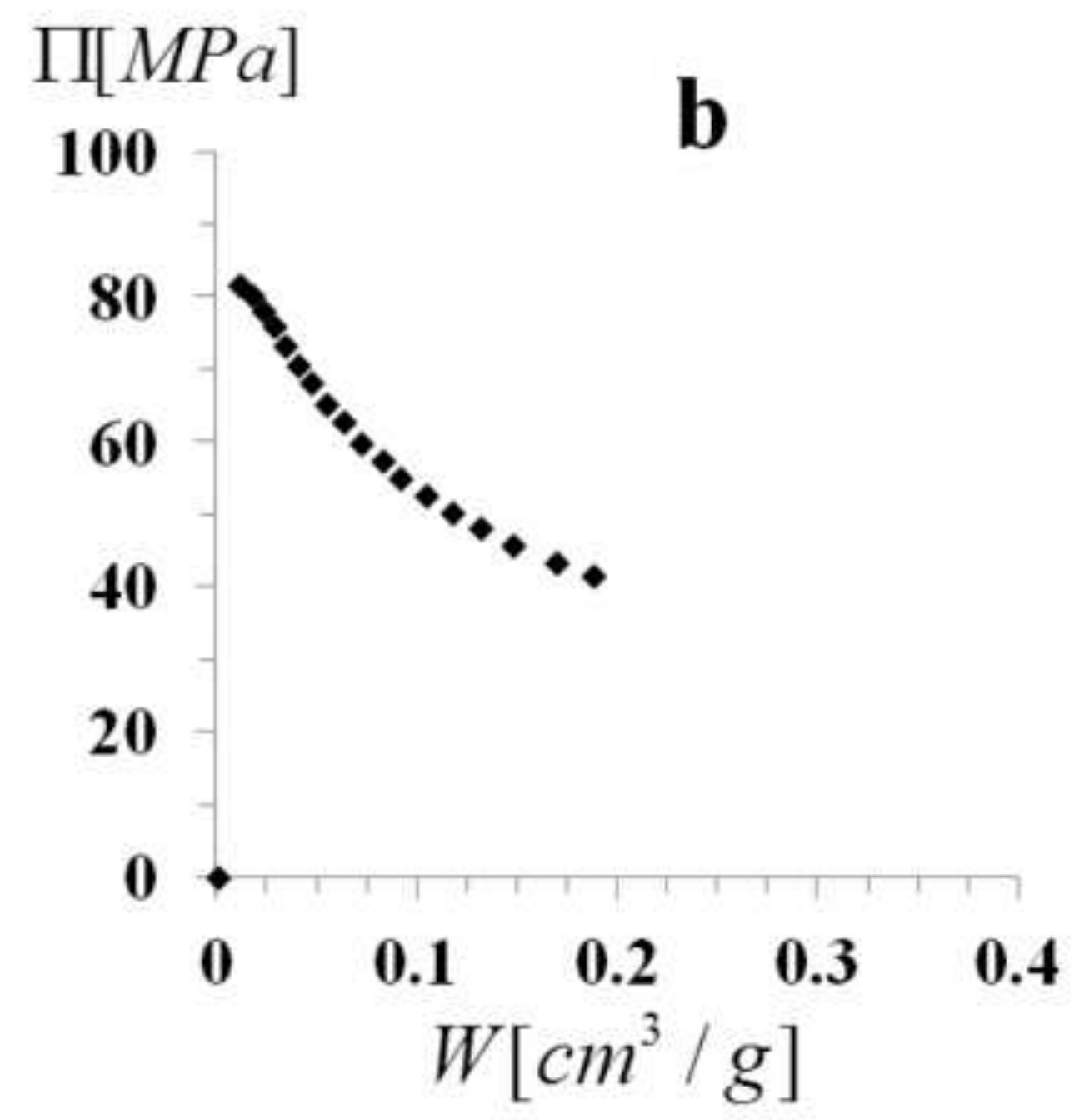


Akaganéites are thermally unstable and superparamagnetic, thereafter the obtained stable and magnetic phase open new applications for these materials. For example, as high temperature catalysts. 